\title{
Introduction to Chronobiology
}

\author{
Sandra J. Kuhlman, ${ }^{1}$ L. Michon Craig, ${ }^{2}$ and Jeanne F. Duffy ${ }^{3}$ \\ ${ }^{1}$ Department of Biological Sciences, and Center for the Basis of Neural Cognition Carnegie Mellon University, \\ Pittsburgh, Pennsylvania 15213 \\ ${ }^{2}$ Division of Sleep and Circadian Disorders, Department of Medicine, Brigham and Women's Hospital and \\ Division of Sleep Medicine, Harvard Medical School, Boston, Massachusetts 02115; and Morehouse School of \\ Medicine, Atlanta, Georgia 30310 \\ ${ }^{3}$ Division of Sleep and Circadian Disorders, Department of Medicine, Brigham and Women's Hospital; and \\ Division of Sleep Medicine, Harvard Medical School, Boston, Massachusetts 02115
}

Correspondence: skuhlman@cmu.edu; jduffy@research.bwh.harvard.edu

A diverse range of species, from cyanobacteria to humans, evolved endogenous biological clocks that allow for the anticipation of daily variations in light and temperature. The ability to anticipate regular environmental rhythms promotes optimal performance and survival. Herein we present a brief historical timeline of how circadian concepts and terminology have emerged since the early observation of daily leaf movement in plants made by an astronomer in the 1700s.

\begin{abstract}
$A$ s a consequence of the Earth's rotation about Aits axis approximately every $24 \mathrm{~h}$, most organisms on this planet are subjected to predictable fluctuations of light and temperature. A diverse range of species, from cyanobacteria to humans, evolved endogenous biological clocks that allow for the anticipation of these daily variations. Thus, our internal physiology and function is fundamentally intertwined with this geophysical cycle. In fact, it was an astronomer, Jean-Jacques d'Ortous de Mairan, rather than a biologist, who provided early insight to this evolutionary relationship between internal physiology and the geophysical cycle. de Mairan (1729) made the observation that daily leaf movements in heliotrope plants continue in constant darkness. To emphasize the endogenous or self-sustained nature of biological
\end{abstract}

clocks, Franz Halberg in 1959 coined the term "circadian" (Latin: $\operatorname{circa}=$ about; dies = day) to refer to daily rhythms that are truly endogenously generated, that is, rhythms having a period of about $24 \mathrm{~h}$ that continue to oscillate in the absence of any environmental input (Chandrashekaran 1998). Rhythm generation is now understood to be an intrinsic property of single cells, driven by an intracellular molecular oscillator based on transcriptional/posttranslational negative feedback loops. Under normal conditions, these endogenous oscillations are synchronized to the environment, and it is generally thought that biological clocks provide an adaptive advantage by ensuring that an organism's internal biochemical and physiological processes, in addition to behavior, are optimally adapted to the local environment.

Editors: Paolo Sassone-Corsi, Michael W. Young, and Akhilesh B. Reddy

Additional Perspectives on Circadian Rhythms available at www.cshperspectives.org

Copyright (C) 2018 Cold Spring Harbor Laboratory Press; all rights reserved; doi: 10.1101/cshperspect.a033613

Cite this article as Cold Spring Harb Perspect Biol 2018;10:a033613 
S.J. Kuhlman et al.

\section{HISTORICAL TIMELINE}

As early as 1729 , it was documented that this daily rhythmic behavior was likely endogenously generated, and it was also near this time that Carl von Linne (1707-1778) constructed a "floral clock" noting the predictability of petal opening and closing times of various species of flowers (Chandrashekaran 1998). However, it was not until 200 years later that Erwin Bünning provided the first evidence for the genetic basis of circadian rhythms generation by demonstrating that period length is heritable in bean plants (McClung 2006). Bünning also put forth an influential hypothesis that circadian oscillators can be used to measure seasonal changes in addition to measuring daily cycles (McClung 2006) and pointed out the adaptive significance of tracking seasonal changes. Thus, the field of circadian rhythms originated from keen observation of plants, and it was not until later that the first observations of endogenously driven rhythms in bacteria (Mitsui et al. 1986), singlecell eukaryotes (Sweeney and Hastings 1957), insects (Beling 1929), birds (Kramer 1952), rodents (Richter 1922), primates (Simpson and Gaibraith 1906), and humans (Aschoff and Wever 1962) were discovered.

A breakthrough in understanding the genetic basis for rhythms generation was made by Ronald Konopka and Seymour Benzer using a mutant screen in Drosophila melanogaster (Konopka and Benzer 1971). Mutagenized flies were examined for the persistence of two circadian behaviors: pupal eclosion and locomotor activity. Flies displayed one of three categorical mutant phenotypes: a lengthening of circadian period, a shortening of period, or arrhythmia. All phenotypes were complemented by a single locus, now referred to as the Period gene. Shortly after this discovery in fruit flies, the frequency gene was shown to be essential for rhythms in conidiation to persist in the filamentous fungus Neurospora crassa (Feldman and Hoyle 1973). These results showed that single gene mutations could disrupt a complex behavior and, together with the discovery of a heritable timing mutation in hamsters by Martin Ralph and Michael Menaker (Ralph and Menaker 1988), provided the rationale for pursuing a large-scale mutant screen in mice (Vitaterna et al. 1994). Subsequently, using a variety of strategies, dozens of clock genes have been discovered in both prokaryotic and eukaryotic systems, including cyanobacteria, fungi, plants, insects, and mammals. Even human rhythms are potently altered by clock gene mutations (Jones et al. 1999). A striking common principle emerges from examining these diverse clock gene systems, that is, all organisms seem to have evolved transcriptional/posttranslational feedback loops to ensure high amplitude, near 24-h, rhythms generation (Fig. 1). Furthermore, the identification of specific clock genes has led to the development of real-time bioluminescent and fluorescent reporters that have the spatial resolution to track rhythmicity at the level of the functional unit of rhythms generation (i.e., the single cell) (Hastings et al. 2005). Such resolution is essential to understand how individual oscillators are coupled within a population of rhythmic cells.

However, in the midst of this clock gene explosion, there is an important lesson to be learned from a pivotal experiment conducted using clock components of cyanobacteria. In 2005, Nakajima and colleagues reconstituted a circadian oscillator in a test tube using only cyanobacterial proteins and ATP (Nakajima et al. 2005). The lesson: it is possible to construct a near 24-h oscillator in the absence of gene transcription. Indeed, circadian research has expanded in recent years to include a systems-level perspective on the interdependencies of cellular metabolic networks such as rhythmic redox cycles and transcription-translation feedback loops (Ray and Reddy 2016).

It is clear from the work of many talented and dedicated scientists that most organisms inherit the innate ability to keep track of time on a 24-h scale. How do organisms use their timing devices? What are the negative consequences if organisms are no longer able to effectively use their clocks, such as in disease or aging? We know from the work of Karl von Frisch and Ingeborg Beling that bees use their clocks to visit flowers at the appropriate time of day so that they can feed when the flower is open. Work 


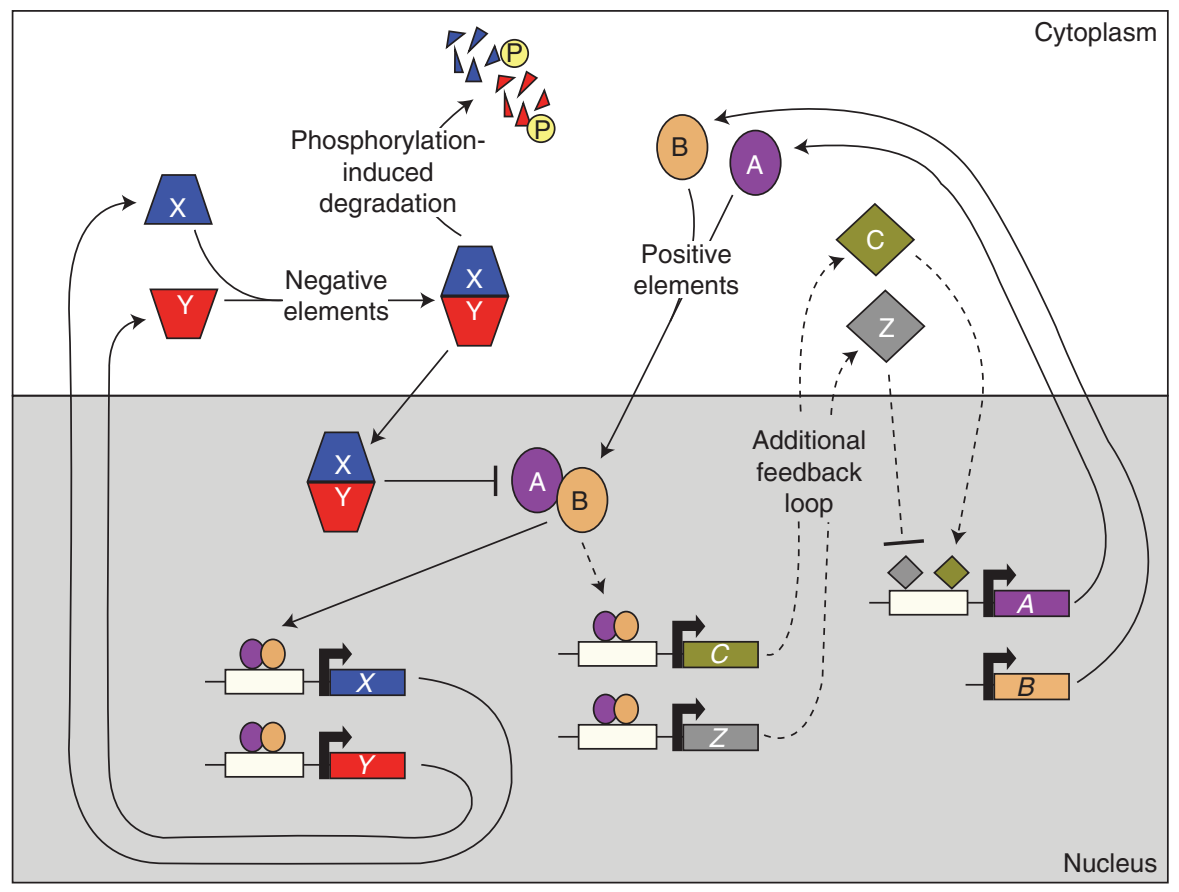

Figure 1. Transcription-translation regulatory feedback loops: a common theme in the generation of eukaryotic circadian rhythms. Many circadian systems are composed of positive and negative elements that are involved in feedback loops. Positive elements (ovals) interact and bind to upstream elements (white rectangles) in the promoter regions (bent arrows) of genes that encode negative elements to activate their expression. These negative components (tetrahedrons) interact to inhibit the activity of the positive elements, which ultimately leads to a decrease in expression of the negative elements. Progressive phosphorylation $(\mathrm{P})$ of negative elements leads to their degradation and a relief of the inhibition of the positive effectors to allow the cycle to start again. Other components (diamonds) may be present that bind to the promoters of genes that encode the positive elements to comprise an additional feedback loop. Arrows and perpendicular lines indicate positive and negative regulation, respectively (see also Bell-Pedersen et al. 2005).

from Gustav Kramer shows that birds use their biological clock during migration to help compensate for the changing position of the sun throughout the day. Additionally, DeCoursey showed that chipmunks use an innate biological clock to properly time foraging so as to avoid predation (DeCoursey et al. 2000), and studies on monarch butterflies revealed that the circadian clock participates in initiating migration by tracking seasonal changes (Reppert et al. 2010). Future studies in the field of circadian rhythms will continue to explore the ways in which organisms use their biological clocks to coordinate internal functions, time their behavior, and navigate through the environment (see Buijs and Kalsbeek 2001 for mammalian review).

\section{CIRCADIAN TERMINOLOGY}

Here we define some commonly used terms in the field. "Black-box" experimental designs are frequently used to probe the mechanisms underlying clock function (Moore-Ede et al. 1982). For example, de Mairan used a black-box approach in his heliotrope plant study. The plant was treated as a system whose internal components were unknown (i.e., black-box), but whose function was studied by assaying the observable output of leaf movement, in response to perturbations caused by environmental inputs (the light-dark cycle). To a large extent, the following terminology developed over the years to precisely report the results of black-box experiments. 
S.J. Kuhlman et al.

Observable, or measurable, output rhythms of the circadian timing system, such as leaf movements, are defined as overt outputs. In the case of animals, wheel-running activity and levels of circulating hormones in the blood are two commonly assayed overt outputs. In addition, a system can be composed of multiple oscillators in which the output signal of one oscillator influences the circadian properties of another oscillator. The output signal can then be referred to as a coupling signal. In practice, the distinction between an overt rhythm and coupling signal may depend on the experimenter's perspective.

A rhythm is considered to be "circadian" if the oscillation has a period of approximately $24 \mathrm{~h}$ and continues in constant conditions, such as constant light (referred to as LL) or constant darkness (DD) (Fig. 2A). The inability of a rhythm to persist under constant conditions implies that the rhythm is driven by responses to periodic external time cues (or a "zeitgeber," German for time-giver) or periodic changes in behavior, rather than generated by a periodic internal process. It is important to keep in mind that when rhythmicity is measured at the tissue or population level, the loss of an overt rhythm owing to experimental perturbation may not be the result of loss of rhythmicity per se. Rather, individual oscillations may continue, but are not observed because of phase differences among oscillating units (Fig. 3).

The time needed for one cycle of a circadian oscillation to occur in constant conditions is known as the free-running period (FRP). Under normal conditions, circadian rhythms are not free-running, rather, they are synchronized to the local environment. The process by which a rhythm synchronizes to an external cycle is referred to as entrainment. Interestingly, although circadian rhythms persist in constant conditions, the FRP can vary slightly in response to changing light intensities. Diurnal organisms display a slightly shorter FRP (i.e., faster clock) in higher light intensities than they do in low light; nocturnal organisms show the inverse response with a longer FRP (i.e., slower clock) in high light than in dim light. This phenomenon, originally described by Jürgen Aschoff, is known as "Aschoffs Rule" (Aschoff and Wever 1962).
FRP can also vary based on the light history that the organism experienced before being placed in constant conditions (Pittendrigh and Daan 1976a; Scheer et al. 2007). The influence of light history on FRP is referred to as an "after effect." Clearly, when assaying overt rhythms and calculating the FRP, the influence of these factors should be taken into consideration.

In addition to being endogenously generated, there are two other properties of circadian rhythms that are often the focus of experimental studies: (1) the ability of the rhythm to be reset, or phase-shifted, by transient exposure to time cues such as light; and (2) temperature compensation, defined below (Pittendrigh 1981a,b; Dunlap et al. 2004).

\section{PHASE-SHIFTING AND THE PHASE-RESPONSE CURVE (PRC)}

The phase of a rhythm can be shifted (or reset) by transient exposure to certain environmental cues (Fig. 2B). Because FRPs are close to, but not exactly $24 \mathrm{~h}$, the rhythm must be reset each day to avoid falling out of sync with the external environmental cycle. As discussed earlier, many organisms use the daily alteration of light and darkness as the environmental signal to entrain their rhythms to the 24-h day. An early study by Hastings and Sweeney (1958) in the dinoflagellate Gonyaulax polyhedra showed a property common among circadian systems: the biological clock is not equally sensitive to light at all times of day. As has been shown subsequently for many organisms that use light as an entraining signal, light exposure in the early subjective night produces a "phase-delay" shift (that is, the rhythm is delayed to a later hour), whereas light exposure in the late subjective night produces a "phase-advance" shift (the rhythm is advanced to an earlier hour), and light during the subjective day produces very small or no phase shifts (Pittendrigh and Daan 1976b). Thus, the response to light is dependent on the organism's internal sense of time. The magnitude and direction of these responses can be plotted with respect to the organism's own circadian phase at which the light was presented, and such a plot is referred to as a PRC (Fig. 2C). 
Introduction to Chronobiology

A
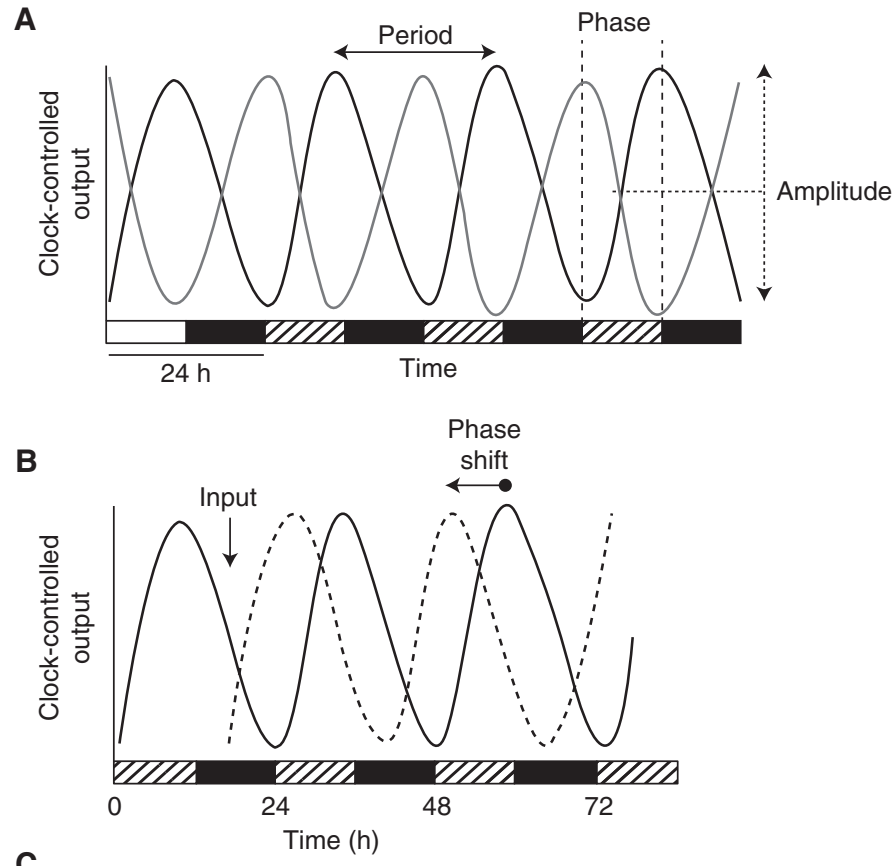

C

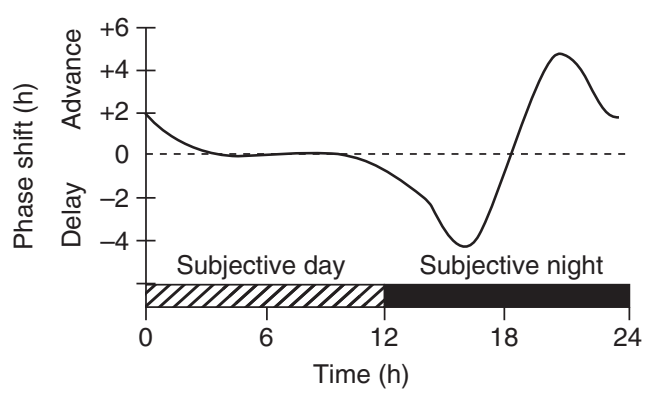

Figure 2. Properties of a circadian rhythm. (A) Behavioral circadian rhythms can be entrained by external stimuli, such as a light-dark cycle, and will persist with a near 24 -h period in the absence of environmental cues, such as constant darkness. Properties of the rhythm that are commonly measured are period, phase, and amplitude. Period is the duration of time to complete one cycle. It is typically measured from peak to peak, but it can be measured from any specific position on the curve. Phase is the relative position on the curve (e.g., the peak) in reference to a particular time, such as time placed in constant darkness. Amplitude is the measurement of the recorded output from the midline of the curve to either the peak or trough. $(B)$ The phase of a circadian rhythm can be reset by the stimuli to which it entrains. In this case, exposure to a stimulus (input) rapidly lowers the level of the rhythmic variable (dashed line), which recovers to a rhythm with a shifted phase as compared with the curve that did not receive the input (solid line). On the $x$-axis time in the light-dark cycle is depicted by alternating white and black bars, and time under constant conditions is depicted by alternating hatched and black bars. Level of clock-controlled output is displayed on the $y$-axis. $(C)$ Phase-response curves measure the magnitude and direction of phase-dependent responses to brief exposures of an external stimulus. The $x$-axis represents the circadian time at which a light pulse is applied to an organism; the $y$-axis shows the change in phase of the circadian-controlled output in response to the light pulse. Positive shifts are indicated as advances and negative phase shifts are delays. A brief light pulse given to an organism during the subjective day (the organism's own internal day) produces little to no phase response, light in the early subjective night (the organism's own internal early night) produces phase-delay shifts, and light in the late subjective night produces phase-advance shifts. 
S.J. Kuhlman et al.

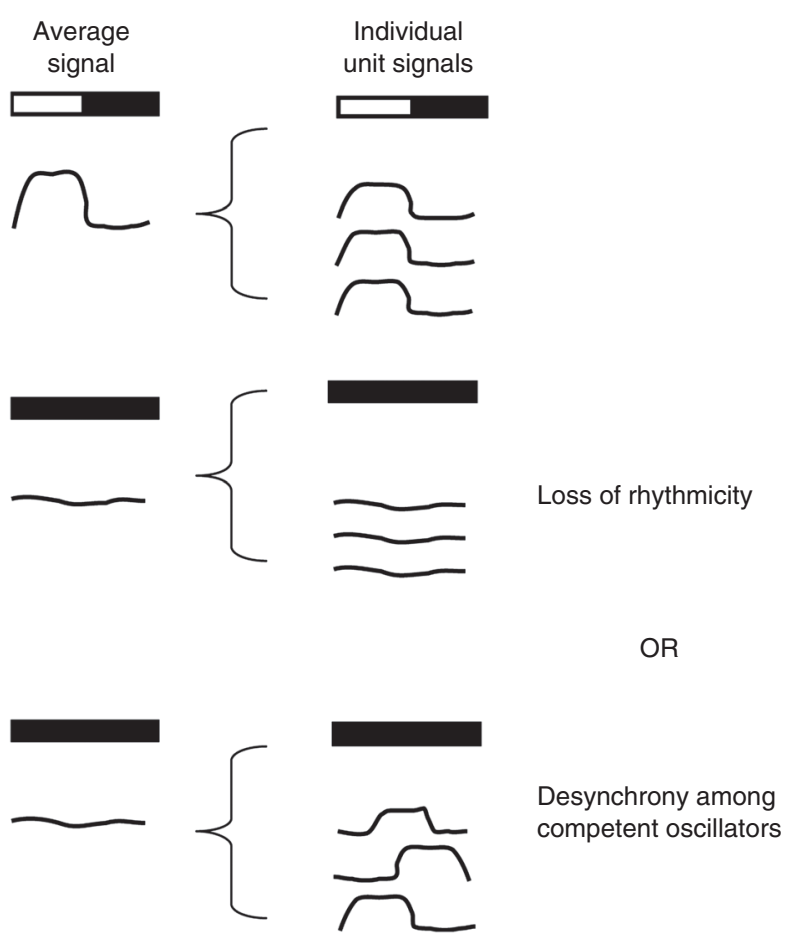

Figure 3. Importance of assaying rhythms at a single-cell resolution. To be considered circadian, a rhythm must be maintained under constant conditions. When examining rhythms at the tissue level in which the tissue is composed of multiple cells, it is important to realize that the absence of rhythmicity under constant conditions could be attributable to two possibilities: either the loss of rhythmicity (indicating the rhythm is not circadian) or desynchrony among oscillators. In the latter case, individual rhythms may actually be circadian; however, the assay has insufficient resolution to show the presence of rhythmicity. To distinguish between the two possibilities, assays sensitive to the functional unit of rhythms generation must be used.

It is important to realize that there are cases in which it is possible that exposure to an external cue will simply transiently perturb the level of the rhythm being assayed, without leading to a persistent shift in the rhythm. This condition is referred to as "masking." Mechanistically, masking can arise because sensory pathways may act on a specific behavior independent of the clock.

\section{TEMPERATURE COMPENSATION}

For a biological clock to be reliable in a natural setting, its period should not change much despite changes in ambient temperature. Morning events should occur at essentially the same time, independent of weather conditions the night before. This property, termed temperature compensation, stems from the observation that the value of the FRP changes very little during exposure to different temperatures within the organism's physiological range. The effect of changes in temperature on the rate of most biochemical reactions is measured by a $\mathrm{Q}_{10}$ value, which is defined as the ratio of the rate of a given process at one temperature to the rate at a temperature $10^{\circ} \mathrm{C}$ lower. The $\mathrm{Q}_{10}$ value of the period of a circadian rhythm remains near 1 , as opposed to other known biochemical reactions that have $Q_{10}$ values of 2 or 3 .

However, temperature compensation is not the same as temperature insensitivity. Temperature itself can be a strong zeitgeber in some organisms (Liu et al. 1998). Individual reactions within the clock are undoubtedly affected by changes in temperature, but the system as a whole is buffered such that the output of rhyth- 
mic behavior does not show large variations in its FRP.

\section{CONCEPT OF A CLOCK}

What is a circadian clock and how has the concept of a "clock" facilitated research? As described in one account (Chandrashekaran 1998), the now widespread use of the term "clock" was in part inspired by Kramer's studies on time-compensation of the sun compass in birds. Shortly thereafter in 1960, the Cold Spring Harbor Symposium of Quantitative Biology was boldly titled "Biological Clocks." Today the term "clock" is used pervasively to emphasize the endogenous nature of rhythm generation, that circadian rhythms are innate rather than learned or passive phenomena, and to imply that a primary function of circadian rhythms is to measure time (Pittendrigh 1960; Moore-Ede et al. 1982). It is this conceptual framework that prompted the design of shifted-schedule or jet-lag experiments and helps us understand the relationship between seasonal adaptation and circadian rhythm generation.

Conceptually, the components of a circadian clock can be broken down into three basic elements: an input pathway, a pacemaker, and an output pathway (Fig. 4). At the heart of a circa- dian clock is the "pacemaker," a central oscillator or a network of coupled oscillators (Welsh et al. 1995) that is entrainable and has the ability to synchronize downstream targets. The temporal information produced by the pacemaker is interpreted by the "output pathways," which then regulate the timing of metabolic and behavioral processes. For the oscillator to maintain in sync with the environment, "input pathways" must relay external timing cues to the pacemaker. Although conceptually one can discuss these three elements as distinct entities, in biological terms, one protein or physiological process can subserve multiple roles.

\section{A CENTRAL PACEMAKER CAN COORDINATE NUMEROUS PERIPHERAL CLOCKS}

Within multicellular organisms, individual cells possess the ability to oscillate on a circadian scale and are themselves bona fide clocks in the sense that their gene transcription-translation feedback loops are entrainable by factors such as internally circulating hormones and that they are capable of producing rhythmic outputs in gene expression and protein function (Schibler and Sassone-Corsi 2002). Typically, the circadian oscillations that exist within an organism are hierarchically arranged and regulated by a dis-

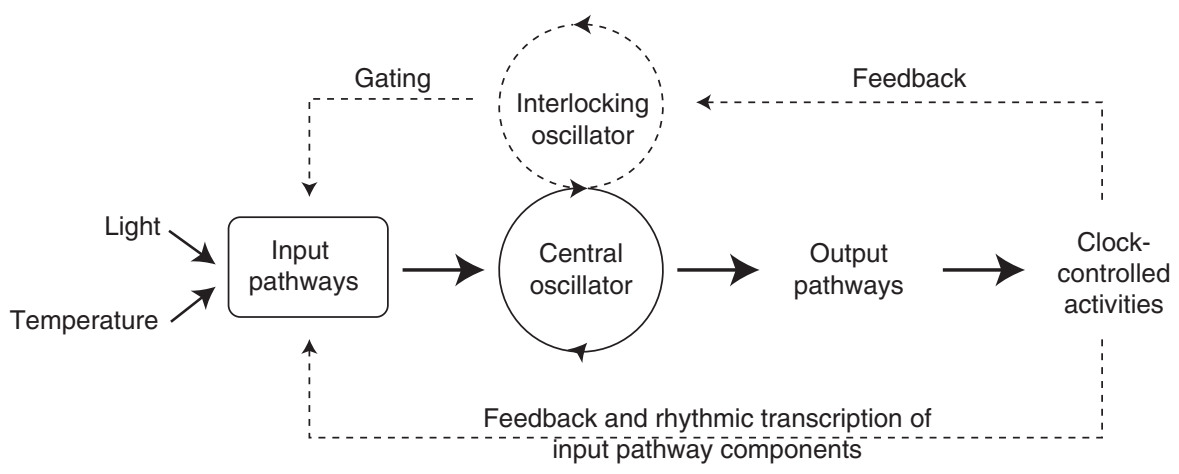

Figure 4. Representation of circadian clock divisions. A circadian clock can be depicted as having input pathways, a central oscillator (or pacemaker), and output pathways. The central oscillator produces the endogenous biological rhythm and can be synchronized with the environment via input pathways through cues such as light or temperature. Output pathways convey the clock's rhythms to downstream targets and drive overtly rhythmic activities. Some circadian systems consist of more elaborate pathways (shown as dashed lines) that include multiple, interlocking oscillators and positive or negative feedback from clock-controlled activities to oscillator and/or input components. (Figure based on data in Gardner et al. 2006.) 


\section{S.J. Kuhlman et al.}

crete identifiable tissue or small group of cells that act as a "central pacemaker" (sometimes referred to as a master pacemaker) capable of maintaining synchrony among the various clocks. Evidence for a hierarchical organization of clocks comes, in part, from inactivation and genetic manipulation experiments. In mammals, inactivation (by lesion, for example) of the suprachiasmatic nucleus (SCN) results in a loss of rhythmic outputs, including the sleepwake cycle, wheel-running behavior, hormone production, core body temperature, and accumulation of clock-controlled messenger RNA (mRNA) and protein at the organ and tissue level (Yamazaki et al. 2000; Moore 2013). Similarly, in Drosophila, genetic ablation experiments established that the ventral lateral neurons (LNvs) are essential for self-sustained behavioral rhythms (Renn et al. 1999). In addition to receiving timing information from a central pacemaker, peripheral clocks are often positioned to receive timing information from other periodic signals, such as luminance level, food intake, or locomotor activity. Thus, although central pacemakers exist, they rarely have exclusive control over the many clocks found within the body of a multicellular organism. Furthermore, the coupling strength among oscillators of the central pacemaker as well as between the central pacemaker and downstream targets can vary, and this variation may promote adaptation to a changing environment, such as day length (Stoleru et al. 2007; Beckwith and Ceriani 2015).

\section{CIRCADIAN TIMING IN HUMANS}

Humans living in industrialized societies with widespread use of artificial light, round-theclock jobs and social activities, and the ability to travel rapidly across time zones are often exposed to conditions in which their internal circadian rhythms become desynchronized from the experienced light-dark cycle. There is now increasing evidence that disruption of internal rhythmicity can have negative health outcomes in people, including insufficient sleep and the corresponding drowsiness and associated safety issues, increased susceptibility to immune challenges, increased risk of cardiovascular and met- abolic disease, and increased cancer risk. These disorders are thought to arise from disruption between the central SCN pacemaker and peripheral clocks attributable to a mismatch between environmental light-dark signals, behavior, and internal oscillations. There is also evidence that desynchronization of individual cells within an organ can occur, leading to inefficient function of that organ (Buijs et al. 2016). As our methods of assessing circadian rhythmicity in humans in the field and clinic improve, the field of chronomedicine will be able to develop, as will chronotherapies to treat circadian desynchrony.

Applying our understanding of the circadian timing system to the treatment of human disease holds huge potential for improving health through personalized medicine. The potential for such therapeutic advances is highlighted by a recent study that found substantial overlap between genes that are rhythmically expressed, genes that are associated with disease, and genes that are targets of the best-selling medications (Fig. 5) (Zhang et al. 2014). More than half of the best-selling medications in the United Sates target genes that oscillate in a circadian manner, and more than 100 of the World Health Organization's (WHO) essential medications target circadian genes, indicating that the pharmacokinetics and pharmacodynamics of those medications are likely to vary with time of day (Dallmann et al. 2016). However, clinical and preclinical drug studies do not typically vary or attempt to optimize the time of day at which medications are administered (Selfridge et al. 2016), leaving open the possibility that efficacy could be improved, side effects could be reduced, or both, if the ideal time of day for administration were known (Ballesta et al. 2017). Moreover, with the development of methods for rapidly and easily assessing circadian timing in the field or clinic, the timing of medication administration will be able to be personalized to account for individual differences in circadian timing (Chang et al. 2016).

\section{CONCLUSION}

In summary, circadian rhythm research has revealed how cellular processes interact to produce 


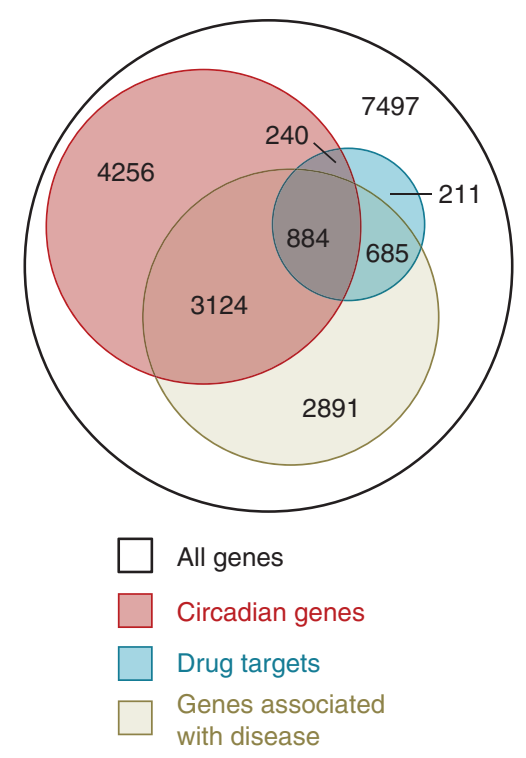

Figure 5. Schematic representation of the overlap between circadian genes and those with important medical relevance. The figure shows the overlap between genes that have a circadian oscillation (red), those known to be associated with disease (tan), and those that are targets of the 100 top-selling drugs in the United States (blue). Few of the drugs are prescribed with specific timing information, representing potential targets for development of chronopharmacology to improve efficacy and/or reduce side effects. (Panel $A$ from Zhang et al. 2014; reprinted, with permission, from the National Academy of Sciences (C) 2014.)

daily rhythms in physiology and behavior that can increase fitness across a diverse range of organisms and species. Three hundred years on, we are now at the cusp of harnessing this knowledge to reveal fundamental properties of cellular networks from a systems biology perspective and to improve human health.

\section{ACKNOWLEDGMENT}

We thank Dr. Shannon R. Mackey for her contributions to the 2007 version of this introduction.

\section{REFERENCES}

Aschoff J, Wever R. 1962. Spontanperiodik des menschen bei ausschluss aller zeitgeber. Naturwissenschaften 49: 337-342.
Ballesta A, Innominato PF, Dallmann R, Rand DA, Lévi FA. 2017. Systems chronotherapeutics. Pharmacol Rev 69: 161-199.

Beckwith EJ, Ceriani MF. 2015. Experimental assessment of the network properties of the Drosophila circadian clock. J Comp Neurol 523: 982-996.

Beling I. 1929. Über das zeitgedächtnis der bienen. Zeitschrift für Vergleichende Physiologie 9: 259-338.

Bell-Pedersen D, Cassone VM, Earnest DJ, Golden SS, Hardin PE, Thomas TL, Zoran MJ. 2005. Circadian rhythms from multiple oscillators: Lessons from diverse organisms. Nat Rev Genet 6: 544-556.

Buijs RM, Kalsbeek A. 2001. Hypothalamic integration of central and peripheral clocks. Nat Rev Neurosci 2: 521526.

Buijs FN, León-Mercado L, Guzmán-Ruiz M, Guerrero-Vargas NN, Romo-Nava F, Buijs RM. 2016. The circadian system: A regulatory feedback network of periphery and brain. Physiology (Bethesda) 31: 170-181.

Chandrashekaran MK. 1998. Biological rhythms research: A personal account. J Biosci 23: 545-555.

Chang AM, Bjonnes AC, Aeschbach D, Buxton OM, Gooley JJ, Anderson C, Van Reen E, Cain SW, Czeisler CA, Duffy JF, et al. 2016. Circadian gene variants influence sleep and the sleep electroencephalogram in humans. Chronobiol Int 33: 561-573.

Dallmann R, Okyar A, Lévi FA. 2016. Dosing-time makes the poison: Circadian regulation and pharmacotherapy. Trends Mol Med 22: 430-445.

DeCoursey PJ, Walker JK, Smith SA. 2000. A circadian pacemaker in free-living chipmunks: Essential for survival? J Comp Physiol A 186: 169-180.

de Mairan J. 1729. Observation botanique. Hist Acad Roy Sci 35-36.

Dunlap JC, Loros JJ, DeCoursey PJ. 2004. Chronobiology: Biological timekeeping. Sinauer Associates, Sunderland, MA.

Feldman JF, Hoyle MN. 1973. Isolation of circadian clock mutants of Neurospora crassa. Genetics 75: 605-613.

Gardner MJ, Hubbard KE, Hotta CT, Dodd AN, Webb AAR. 2006. How plants tell time. Biochem J 397: 15-24.

Hastings JW, Sweeney BM. 1958. A persistent diurnal rhythm of luminescence in Gonyaulax polyedra. Biol Bull 115: 440-458.

Hastings MH, Reddy AB, McMahon DG, Maywood ES. 2005. Analysis of circadian mechanisms in the suprachiasmatic nucleus by transgenesis and biolistic transfection. Methods Enzymol 393: 579-592.

Jones CR, Campbell SS, Zone SE, Cooper F, DeSano A, Murphy PJ, Jones B, Czajkowski L, Ptáček LJ. 1999. Familial advanced sleep-phase syndrome: A short-period circadian rhythm variant in humans. Nat Med 5: 1062-1065.

Konopka RJ, Benzer S. 1971. Clock mutants of Drosophila melanogaster. Proc Natl Acad Sci 68: 2112-2116.

Kramer G. 1952. Experiments on bird orientation. Int J Avian Sci 94: 265-285.

Liu Y, Merrow M, Loros JJ, Dunlap JC. 1998. How temperature changes reset a circadian oscillator. Science 281: 825-829. 
S.J. Kuhlman et al.

McClung CR. 2006. Historical perspective essay. Plant circadian rhythms. The Plant Cell 18: 792-803.

Mitsui A, Kumazawa S, Takahashi A, Ikemoto H, Cao S, Arai T. 1986. Strategy by which nitrogen-fixing unicellular cyanobacteria grow photoautotrophically. Nature 323: 720 722.

Moore RY. 2013. The suprachiasmatic nucleus and the circadian timing system. Prog Mol Biol Transl Sci 119: 1-28.

Moore-Ede MC, Sulzman FM, Fuller CA. 1982. The clocks that time us. Harvard University Press, Cambridge, MA.

Nakajima M, Imai K, Ito H, Nishiwaki T, Murayama Y Iwasaki H, Oyama T, Kondo T. 2005. Reconstitution of circadian oscillation of cyanobacterial KaiC phosphorylation in vitro. Science 308: 414-415.

Pittendrigh CS. 1960. Circadian rhythms and the circadian organization of living systems. Cold Spring Harb Symp Quant Biol 25: 159-184.

Pittendrigh CS. 1981a. Circadian systems: General perspective. In Handbook of behavioral neurobiology: Biological rhythms (ed. Aschoff J), pp. 57-80. Plenum, New York.

Pittendrigh CS. 1981b. Circadian systems: Entrainment. In Handbook of behavioral neurobiology: Biological rhythms (ed. Aschoff J), pp. 95-124. Plenum, New York.

Pittendrigh CS, Daan S. 1976a. A functional analysis of circadian pacemakers in nocturnal rodents. I: The stability and lability of spontaneous frequency. J Comp Physiol A 106: 223-252.

Pittendrigh CS, Daan S. 1976b. A functional analysis of circadian pacemakers in nocturnal rodents. II: The variability of phase response curves. J Comp Physiol A 106: 253 266.

Ralph MR, Menaker M. 1988. A mutation of the circadian system in golden hamsters. Science 241: 1225-1227.

Ray S, Reddy AB. 2016. Cross-talk between circadian clocks, sleep-wake cycles, and metabolic networks: Dispelling the darkness. Bioessays 38: 394-405.

Renn SC, Park JH, Rosbash M, Hall JC, Taghert PH. 1999. A pdf neuropeptide gene mutation and ablation of PDF neurons each cause severe abnormalities of behavioral circadian rhythms in Drosophila. Cell 99: 791-802.
Reppert SM, Gegear RJ, Merlin C. 2010. Navigational mechanisms of migrating monarch butterflies. Trends Neurosci 33: 399-406.

Richter CP. 1922. A behavioristic study of the activity of the rat. Comp Psychol Monogr 1: 1-55.

Scheer FAJL, Wright KP Jr, Kronauer RE, Czeisler CA. 2007. Plasticity of the intrinsic period of the human circadian timing system. PLoS ONE 2: e721.

Schibler U, Sassone-Corsi P. 2002. A web of circadian pacemakers. Cell 111: 919-922.

Selfridge JM, Gotoh T, Schiffhauer S, Liu JJ, Stauffer PE, Li A, Capelluto DGS, Finkielstein CV. 2016. Chronotherapy: Intuitive, sound, founded... but not broadly applied. Drugs 76: 1507-1521.

Simpson S, Gaibraith JJ. 1906. Observations on the normal temperature of the monkey and its diurnal variation, and on the effect of changes in the daily routine on this variation. Earth Environ Sci Trans R Soc Edinb 45: 65-106.

Stoleru D, Nawathean P, Fernández MP, Menet JS, Ceriani MF, Rosbash M. 2007. The Drosophila circadian network is a seasonal timer. Cell 129: 207-219.

Sweeney BM, Hastings JW. 1957. Characteristics of the diurnal rhythm of luminescence in Gonyaulax polyedra. J Cell Comp Physiol 49: 115-128.

Vitaterna MH, King DP, Chang AM, Kornhauser JM, Lowrey PL, McDonald JD, Dove WF, Pinto LH, Turek FW Takahashi JS. 1994. Mutagenesis and mapping of a mouse gene, Clock, essential for circadian behavior. Science 264: 719-725.

Welsh DK, Logothetis DE, Meister M, Reppert SM. 1995. Individual neurons dissociated from rat suprachiasmatic nucleus express independently phased circadian firing rhythms. Neuron 14: 697-706.

Yamazaki S, Numano R, Abe M, Hida A, Takahashi R, Ueda M, Block GD, Sakaki Y, Menaker M, Tei H. 2000. Resetting central and peripheral circadian oscillators in transgenic rats. Science 288: 682-685.

Zhang R, Lahens NF, Balance HI, Hughes ME, Hogenesch JB. 2014. A circadian gene expression atlas in mammals: Implications for biology and medicine. Proc Natl Acad Sci 111: 16219-16224. 


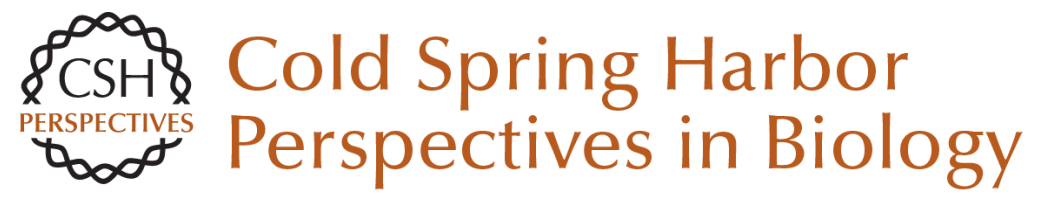

\section{Introduction to Chronobiology}

Sandra J. Kuhlman, L. Michon Craig and Jeanne F. Duffy

Cold Spring Harb Perspect Biol 2018; doi: 10.1101/cshperspect.a033613 originally published online October 16, 2017

\section{Subject Collection Circadian Rhythms}

\section{Circadian Posttranscriptional Regulatory}

Mechanisms in Mammals Carla B. Green

Design Principles of Phosphorylation-Dependent Timekeeping in Eukaryotic Circadian Clocks Koji L. Ode and Hiroki R. Ueda

Interplay between Microbes and the Circadian Clock

Paola Tognini, Mari Murakami and Paolo Sassone-Corsi

\section{A 50-Year Personal Journey: Location, Gene \\ Expression, and Circadian Rhythms Michael Rosbash}

Regulating the Suprachiasmatic Nucleus (SCN)

Circadian Clockwork: Interplay between

Cell-Autonomous and Circuit-Level Mechanisms Erik D. Herzog, Tracey Hermanstyne, Nicola J. Smyllie, et al.

Systems Chronobiology: Global Analysis of Gene Regulation in a 24-Hour Periodic World Jérôme Mermet, Jake Yeung and Felix Naef
Coordination between Differentially Regulated Circadian Clocks Generates Rhythmic Behavior Deniz Top and Michael W. Young

Introduction to Chronobiology Sandra J. KuhIman, L. Michon Craig and Jeanne F. Duffy

Cellular Timekeeping: It's Redox o'Clock Nikolay B. Milev, Sue-Goo Rhee and Akhilesh B. Reddy

Molecular Mechanisms of Sleep Homeostasis in Flies and Mammals Ravi Allada, Chiara Cirelli and Amita Sehgal

Membrane Currents, Gene Expression, and Circadian Clocks Charles N. Allen, Michael N. Nitabach and Christopher S. Colwell

The Plant Circadian Clock: From a Simple Timekeeper to a Complex Developmental Manager Sabrina E. Sanchez and Steve A. Kay

For additional articles in this collection, see http://cshperspectives.cshlp.org/cgi/collection/

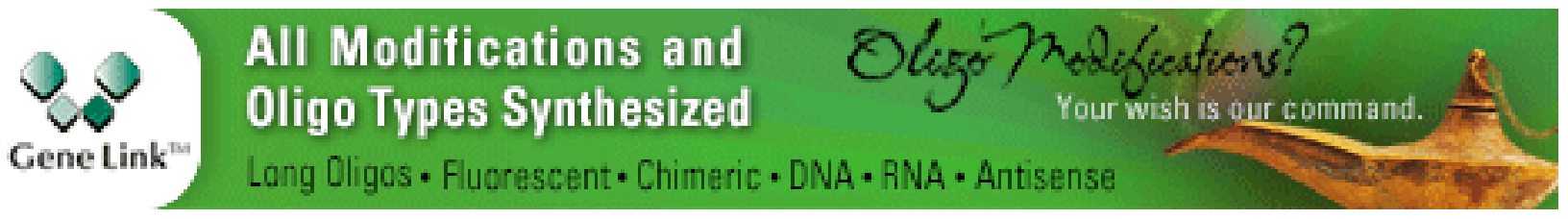

